\title{
Brain-derived Neurotrophic Factor: a Key Gene Risk Factor and Potential Therapy Target in Bipolar Disorder
}

\author{
Yue Zeng ${ }^{1, *}{ }^{*}$, Yutao Chen ${ }^{1, \dagger}$, Juming $\mathrm{Li}^{2, \dagger}$ and Yuqi $\mathrm{Su}^{3, \dagger}$ \\ ${ }^{1}$ School of Life Sciences, Sun Yat-sen University, Guangzhou, Guangdong, China. \\ ${ }^{2}$ College of Life Sciences, Northwest A\&F University, Xianyang, Shanxi, China. \\ ${ }^{3}$ International Department, Zibo Experimental High School, Zibo, Shandong, China. \\ †Those authors contributed equally.
}

\begin{abstract}
Bipolar Disorder (BD) is a worldwide, multifactorial mental disorder characterized by manic and depressive symptoms of varying degrees. Among all the genetic risk factors correlated with BD, brain-derived neurotrophic factor (BDNF) has emerged as a crucial neutropin that influences BD susceptibility with strong conservative across species and multiple downstream signaling pathways. However, the mechanisms of how BDNF polymorphism can contribute to BD are not yet lucid and systematically reviewed. BDNF Val66Met variant is capable of inducing neurodegenisis and Long-term Depression (LTD), both of which account for pathogenesis in BD. The Val66Met variant is associated with rapid cycling episodes in BD. Another variant, Arg125Met is a potential BD risk variant which elicits neuronal apoptosis by affecting the maturation of BDNF. In this paper, we briefly summarized BD epidemiology, symptoms, BDNF structure, and its action of function. We reviewed various mechanisms of BDNF Val66Met and Arg125Met variant for BD pathogenesis in detail and provided insights into possible BD clinical treatment targets. BDNF has been proven to be a noteworthy gene factor in BD and gene therapy targeted on BDNF is a promising therapeutic strategy that requires further research.
\end{abstract}

\section{Introduction}

Bipolar disorder (BD) is a multifactorial disease associated with severe neuropsychological defects, emotional disorders, and even immune and physiological changes [1]. BD can be divided into two subtypes: BD I and BD II. Compared with BD II, BD I is characterized by more intense and destructive general manic episodes, though both types show similar severe depressive symptoms [2]. Accumulating evidence shows that BD is related to genetic factors, developmental defects and gene-environment interaction [3]. BD patients generally suffer from Neurological changes, Immune disorders, neuroplasticity and neurotrophin signaling changes and other pathological alterations4. Changes in neurotrophin levels in particular, such as glial cell derived neurotrophie factor (GDNF), BDNF, serum neurotrophin-3 and neurotrophin-4 were observed in the central nervous system $(\mathrm{CNS})$ of BD patients $[4,5]$.

Among all the neurotrophin factors that influence the susceptibility of BD, BDNF has emerged as a crucial neurotrophin involving in mood disorders. BDNF is extensively expressed in the mammalian brain [6], regulating critical events in cognition and memory, such as formation of new synapses, dendritic branching, and synaptic plasticity changes [7]. Phylogenic analysis shows high sequence conservation in BDNF [8]. BDNF and $\mathrm{m}-\mathrm{BDNF}$ interact with different types of receptors on synapses, activating a wide range of essential signaling pathways in brain development [9].

It has been well established that serum BDNF levels are negatively correlated with $\mathrm{BD}$ episodes and their severity [5]. BDNF crosses the blood-brain barrier and can be detected in serum and plasma [10]. Converging lines of research have proved peripheral BDNF's potential to be a biomarker indicating BD status [11]. Given that BDNF levels are indicative of BD symptoms, the correlation between BDNF polymorphism and BD has also been intensively studied. Among different BDNF variants, the Val66Met SNP (rs6265) has been most widely investigated, though its association with the susceptibility of BD is not concrete as various lines of research contradicts each other [12]. However, the influence of other variants of BDNF on the susceptibility of $\mathrm{BD}$ remains unclear.

An emerging technology, clustered regularly interspaced short palindromic repeats (CRISPR)-Cas9 system exerts an extraordinary utility of editing target genes providing an opportunity for rescuing the mutated site of BDNF and regular signaling pathways [13]. Here, we provide an overview of BD symptoms, BD susceptibility gene BDNF, and an innovative therapy (CRISPR gene therapy) based on existing work.

*Corresponding author: zengy97@mail2.sysu.edu.cn 


\section{$2 \mathrm{BD}$ is a worldwide, multifactorial mental disorder with varying symptoms}

\subsection{Epidemiology}

A large survey of 11 countries showed that the lifetime prevalence rate of bipolar disorder was $2.4 \%$, of which BD I was $0.6 \%$ and BD II $0.4 \%$. The average age of onset of bipolar disorder is generally considered to be about 20 years old [14]. In terms of gender differences, the results are also different: some studies believe that the incidence is roughly the same between different genders [15], while other studies believe that the prevalence rate of BD I in men is higher while that women is more likely to suffer from BD II [14].

\subsection{Symptoms}

The symptoms of bipolar disorder are complex. DSM-5 diagnostic criteria divide BD into a series of diagnostic subgroups according to the severity of mood elevation during acute episodes. BD I has features including inflated self-esteem, decreased need for sleep, pressured speech, racing thoughts, distractibility, psychomotor agitation, and risky behavior that leads to significant functional impairment. The characteristics of BD II are between the two, and the duration and severity are not enough to cause severe functional impairment and insanity, but BD II individuals still experience threshold depressive episodes [16]. The specific diagnostic criteria for DSM-5 are shown in Table 1 [17].

Table 1. DSM-5 specifiers for bipolar and related disorders [17]

\begin{tabular}{cccc}
\hline Specifier & $\begin{array}{c}\text { Manic } \\
\text { episode }\end{array}$ & $\begin{array}{c}\text { Depressive } \\
\text { episode }\end{array}$ & $\begin{array}{c}\text { Ilness } \\
\text { course }\end{array}$ \\
\hline Anxious distress & $\times$ & $\times$ & \\
Mixed features & $\times$ & $\times$ & $\times$ \\
Rapid cycling & & $\times$ & \\
Melancholic \\
features
\end{tabular}

\subsection{Risk Factors of BD}

Many factors are considered to be related to the occurrence of $\mathrm{BD}$, that is, the "risk factor" of $\mathrm{BD}$. These factors include genetic factors and environmental factors. Genetic risk factors (such as BDNF, SNPs) are considered to be one of the important influencing factors of BD. Environmental factors (social stress, childhood trauma, etc.), such as T. gondii, a contagious parasite, is also thought to be associated with the occurrence of BD [18]. Table 2 outlines the various risk factors related to BD.

Table 2. Risk factors of BD [3]

\begin{tabular}{cc}
\hline Genetic Factors & Environmental Factors \\
\hline Multiple SNPs & T. gondii \\
BDNF Val66Met Polymorphism & Childhood Trauma \\
CACNA1C mutation & Psychological stressors \\
COMT mutation & Substance misuse \\
\hline
\end{tabular}

Among all these risk factors, BDNF is noteworthy. It has been confirmed in genetic studies that the diversity of its gene coding is related to BD, and BDNF Val66Met Polymorphism has also been found to have geneenvironment interaction with childhood trauma, which can induce BD to certain extent.

\subsection{Pathophysiology}

Neuroimaging studies of patients with BD or autopsies of patients' cadavers have revealed abnormalities in multiple regions of the brain, the most common areas of which include the ventral prefrontal cortex and the amygdala, where disorders of emotional circuits are thought to be a mechanism of BD [19]. In addition, a series of studies have shown that abnormal nerve transmission, abnormal intracellular signal pathway and abnormal cellular function may all play a role in BD [20].

The clinical symptoms of BD are not directly related to the structural and functional changes of specific brain regions, but have a stronger correlation with the dysfunction of brain neural networks related to emotion, cognition, behavior, neuroendocrine and circadian rhythm [21]. Compared with healthy people, the composition of complex prefrontal-ACC-pallido-striatal-thalamicamygdala network in patients with $\mathrm{BD}$ has undergone structural and functional changes (Figure 1) [21]. 


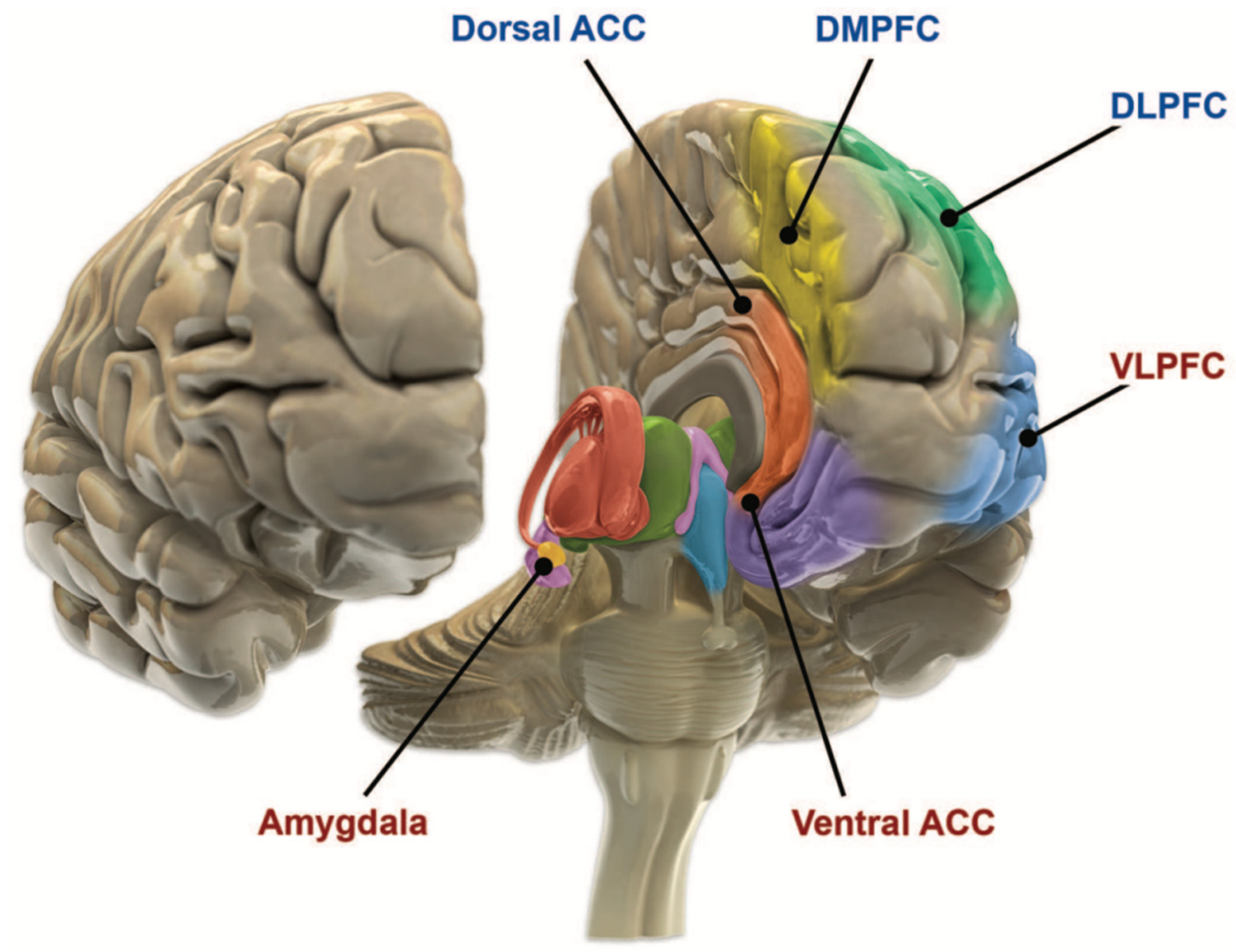

\section{DLPFC: Dorsolateral prefrontal cortex} VLPFC: Ventrolateral prefrontal cortex

\section{DMPFC: Dorsomedial prefrontal cortex ACC: Anterior cingulate cortex}

Fig 1. Functional brain changes in BD [21]. Based on Langan and McDonald. Illustration courtesy of: Roland Tuley, Fire and Rain. Imaging studies of euthymic bipolar patients provide evidence of compromised cognitive control, combined with increased responsiveness of limbic and para-limbic brain regions involved in emotional regulation. Brain areas associated with cognitive control, which manifest reduced responsiveness, are labeled blue (dorsal ACC, DMPFC, and DLPFC). By contrast, limbic and paralimbic brain areas involved in emotional regulation, associated with greater responsiveness, are labeled in red (amygdala, VLPFC, and ventral ACC).

In addition, Previous clinical studies have shown that the level of the brain-derived neurotrophic factor (BDNF) in patients with $\mathrm{BD}$ is low [4], and the low level of BDNF is related to the severity of depression and mania in patients with $\mathrm{BD}$. In addition, drug trials showed that the level of BDNF in patients with manic BD increased significantly after drug treatment [22]. BDNF, known as the Brain-derived neurotrophic factor, is one of the member of the neurotrophin family of secreted proteins. BDNF is associated with synaptic plasticity, neurogenesis, neuronal survival and protection against brain insults in the human brain [23]. There is also evidence of changes in BDNF levels in diseases such as multiple sclerosis and Alzheimer's disease [24]. In view of the important role of BDNF in the brain and as a factor affects BD to a great extent, we decided to use BDNF as the entry point for further discovery on BD and BD's potential treatment.

\section{The Role of Brain-derived Neurotrophic Factor (BDNF) in BD Pathology}

Converging lines of research and cumulating evidence prove the relevance of BDNF and BD. The BDNF gene resides at cytogenetic band $11 \mathrm{p} 13$ on the short arm of chromosome 11 [25]. Studies of samples from the National Institute of Mental Health Genetics Initiative bipolar pedigrees prove linkage evidence in the 11p13p15 On molecular level, studies have demonstrated that compared to healthy controls, BD patients' peripheral BDNF levels are reduced during manic and depressive episodes [26]. Furthermore, BDNF levels negatively correlate with the severity of both manic and depressive symptoms26. In correspondence with the level of protein, $\mathrm{BD}$ patients also have lower level of BDNF mRNA compared to healthy control group [27].

\subsection{BDNF Gene}

The conservative structure of BDNF decides its physiological functions, which is greatly independent of the phylogenetic development stage. The BDNF peptide is a highly conserved chain composed of 247 amino acids. Among vertebrates' and humans' genes, the extent of identity is from $85.9 \%$ to $100 \%$ [28]. To date, there have been several BD animal models. They can be classified into four categories: pharmacological models, nutritional models, environmental models, and genetic models. The effects of levels of BDNF, the BD-associated gene, have also been investigated in animal models. Heterozygous BDNF mice show weight gain, anxiety, aggressiveness, 
and contextual dysmnesia, therefore these consequences have been suggested as an animal model for mood disorders [29]. The establishment of these animal models can further serve as an illustration of conservation.

BDNF is first synthesized as a precursor pro-BDNF, a chain of 247 amino acids, consisting of a pro-domain and a mature domain. The precursor produces mature BDNF
(14 kDa) through $\mathrm{N}$-terminal cleavage within the transGolgi apparatus network and/or immature secretory vesicles. The protein is cleaved in the endoplasmic reticulum by pro-BDNF. The $\mathrm{m}-\mathrm{BDNF}$ then cleavages in intracellular vesicles, allowing this neurotrophin to be released out of the cell via axon terminal and presynaptic membrane [9] (Fig.2).

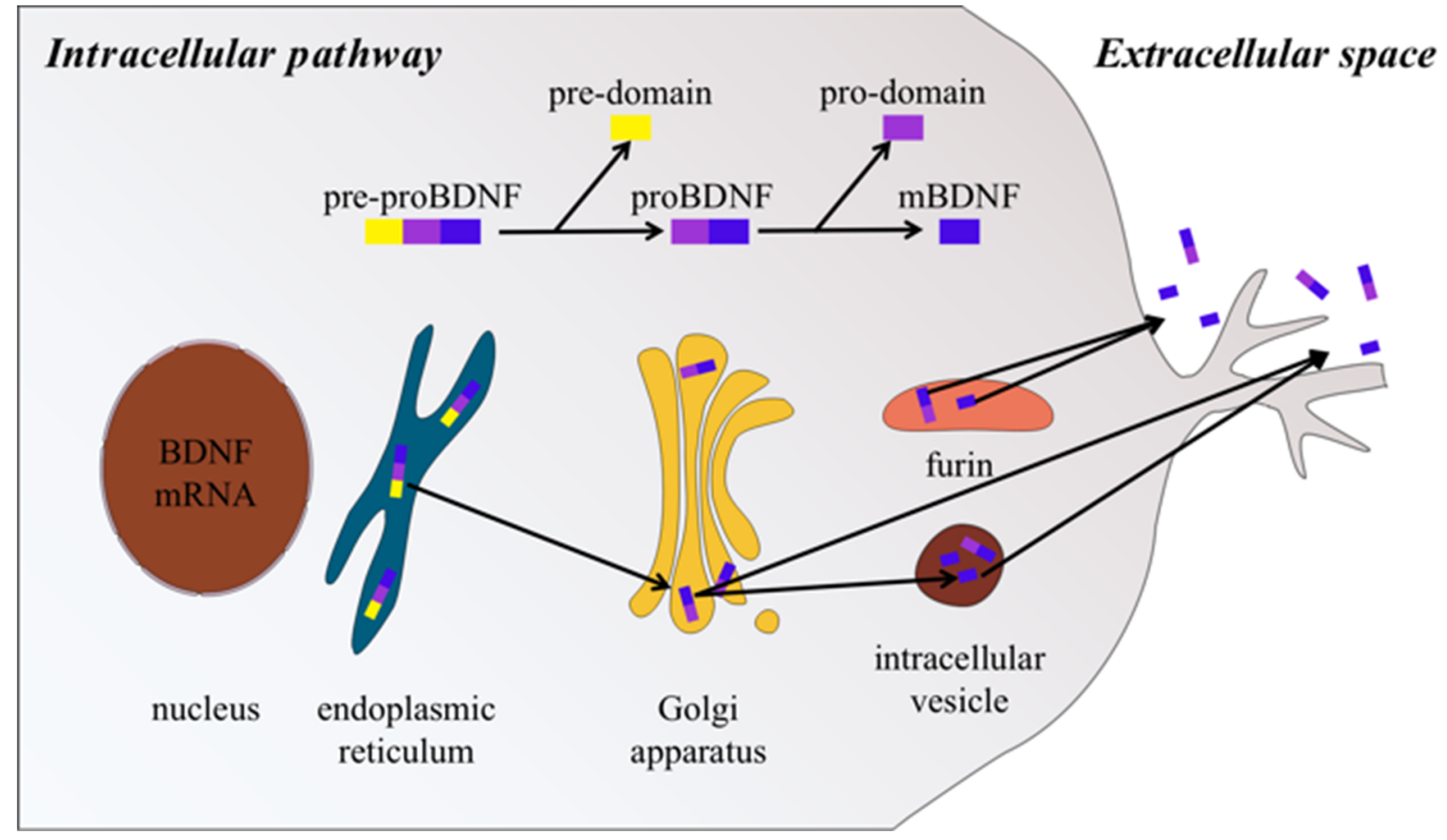

Fig 2. Synthesis and release of BDNF [9]. Pre-proBDNF is synthesized, comprising pro-domain and m-domain, which cleavages into proBDNF. ProBDNF cleavages into mBDNF in Golgi apparatus and/or intracellular vesicle, which is then released into extracellular space through axon terminal and presynaptic membrane.

BDNF controls in a wide variety of processes, involving triggering different signaling pathways by combining with diverse receptors. Thus the pro-BDNF formed from pro-domain and mature domain (m-BDNF) can function in prohibiting or supporting neuronal survival [30]. The mature domain of pro-BDNF interacts with p75 neurotrophin receptor (p75NTR) or sortilin receptor. The pro-domain interacts with sortilin receptor vacuolar protein sorting 10 protein (Vps10p) [28]. Particular complexities formed within cell membrane which are needed to activate receptor and the activated receptor can trigger several signaling pathways [31]. When pro-BDNF, p75NTR or sortlin complex bind with Ras homolog gene family member A (RhoA), the RhoA related pathways can regulate the development and motility of neuronal growth cone; with nuclear factor kappa $\mathrm{B}(\mathrm{NF}-\kappa \mathrm{B})$, the triggered pathways can support neuronal to survive and maintain sufficient number of the neuronal when the brain develops; with c-Jun amino terminal kinase (JNK), the activated JNK related pathways trigger neuronal apoptosis [32]. Other signaling pathways such as PKC-related pathway and PI3K/Aktrelated pathway are able to increase the synaptic plasticity [32] (Fig. 3). In a word, BDNF plays an important role in brain development and synaptic plasticity. These functions are achieved through triggering signaling pathways brought by the interaction between domain of BDNF and different types of receptors [9]. 


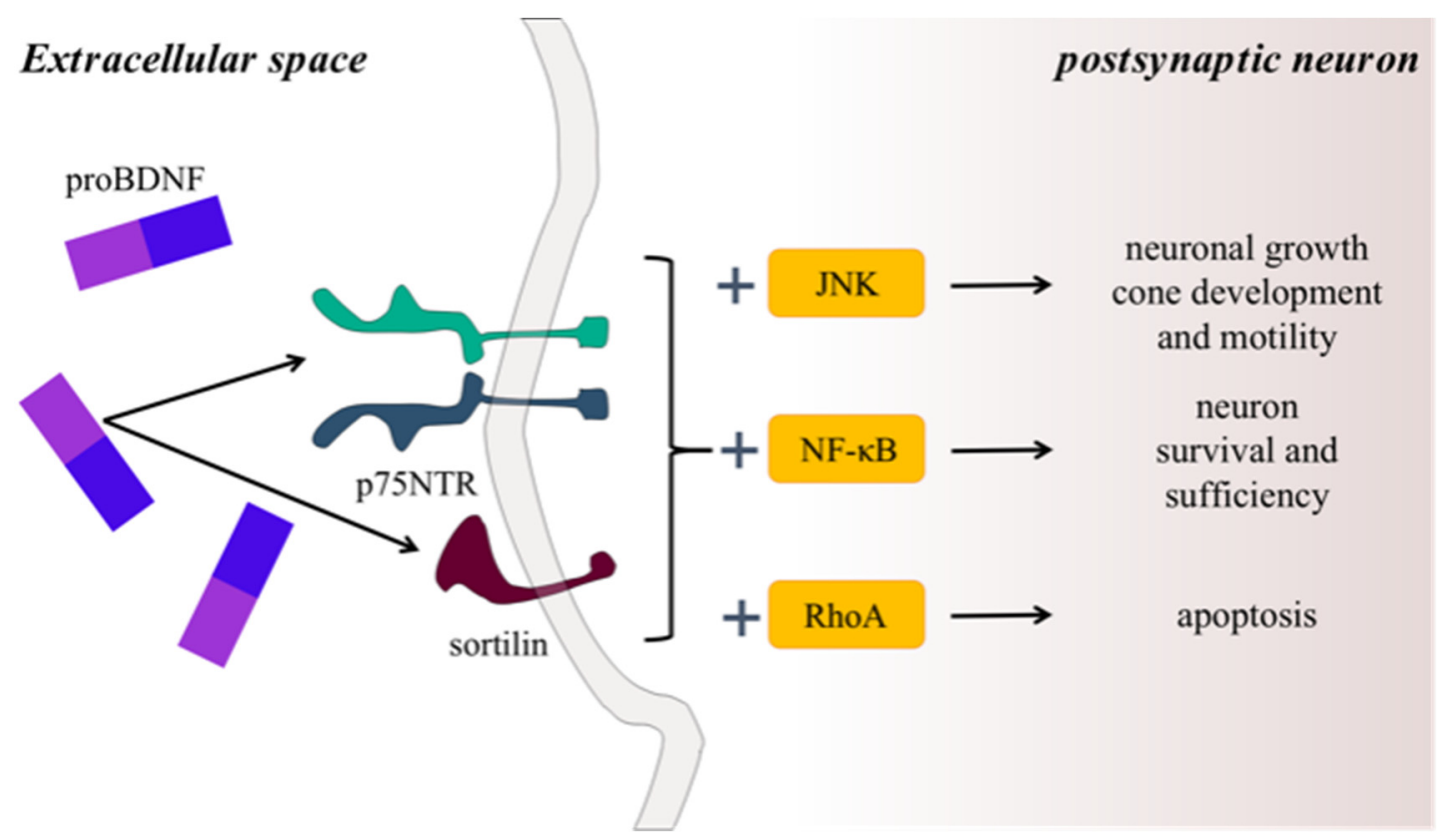

Fig 3. Pro-BDNF interacting with different receptors and triggering different pathways by binding with RhoA/NF- $\mathrm{B}$, JNK[9]. When proBDNF, p75NTR, sortlin bind with JNK, NF- $\mathrm{BB}$ and RhoA respectively, the triggered signaling pathways can control neuronal growth cone development, neuron survival and sufficiency, and apoptosis.

\subsection{Mechanisms of BDNF variants for BD pathogenesis}

Although BDNF is highly conserved across species in the protein sequence, there are enormous number of singlenucleotide polymorphisms (SNPs) in its gene. In dbSNP, the NCBI database of genetic variation, over 40,000 variant entries, most resided in the noncoding sequence, has been recorded for the human BDNF gene. By far, only a small pool of BDNF gene variants are coded with the frequency $>1 \%$, including Val66Met, Arg125Met, Thr2lle, GIn75His, and Arg127Leu (Fig. 4) [33]. These major SNPs reside in the pro-domain of BDNF, leading to a disturbed quantity balance between m-BDNF and pro-BDNF in neuron cells. However, only the Val66Met variant has been studied widely, whereas the ramifications of the rest remain largely obscure.

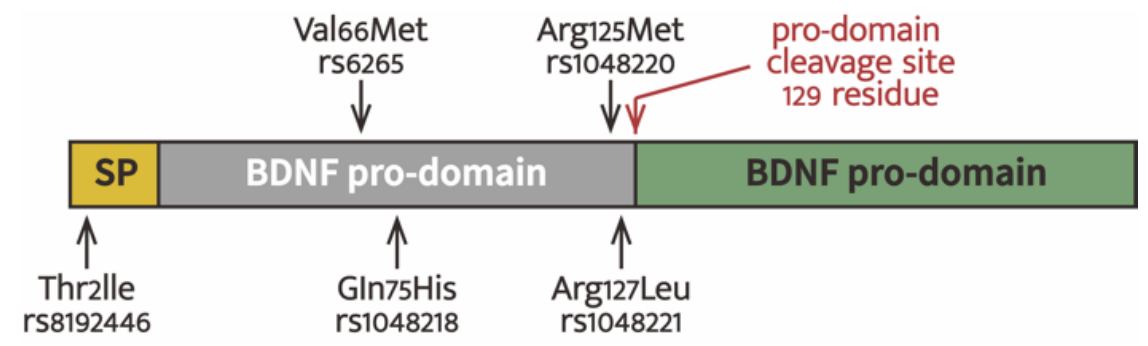

Fig 4. Common SNPs in the BDNF gene[34]. Common BDNF SNPs reside in the BDNF prodomain sequence. The pro- to mature cleavage site is at amino acid residue 129. Data adapted from Uniprot.

\subsection{Val66Met}

The Val66Met polymorphism refers to the amnio acid residue substitution caused by naturally occurring polymorphism from valine (Val) to methionine (Met) At position 66 in the protein sequence of BDNF. Lines of population genetic study demonstrates that $30 \%$ to $50 \%$ of Caucasian populations, and $65 \%$ to $70 \%$ of east Asian populations are made up of Met carriers. The polymorphism is harbored in the BDNF pro-domain, and earliest study of the BDNF Val66Met polymorphism showed that it interferes with the activity-dependent BDNF release [35]. Now increasing evidence adds to the understanding of this BDNF variant. Hence, proposed mechanisms of the Val66Met polymorphism for BD pathogenesis will be reviewed in the following section.

Emerging evidence shows that BD can be considered as a neurodegenesis disorder. BD patients' imaging studies indicates that the disease coincides with neuronal atrophy. Reduced activity in the subgenual prefrontal cortex has been detected during the bipolar depression, together with decreased mean volume on grey matters, and cortical volume [36]. Therefore, neuronal loss could be a cause of BD. Val66Met polymorphism is correlated to the shrinkage of hippocampal volume. An Australian imaging, biomarkers and lifestyle study over 36 months in 165 healthy adults suggests that Met carriers showed a significant reduced volume of hippocampus, compared to the Val/Val homozygote group [37]. Moreover, this 
atrophy is also accompanied with, reduced hippocampal activity and impairments in episodic memory [37]. The Val66Met variant is also associated with and decreased prefrontal cortical volume [38]. knock-in BDNF met/met mice exhibit decreased hippocampal volume in the dentate gyrus [39]. Val66Met polymorphism can induce growth cone retraction as well. They are rich in actin whose extension and retraction are regulated by Rho GTPases family members including Rac [40]. Anastasia et al. demonstrated that the BDNF pro-domain or proBDNF with the Val66Met polymorphism significantly decreases the Rac activity in hippocampal neurons in vitro [31]. Met66 pro-domain possesses an increased helical tendency compared with the Val66 pro-domain, which is favorable in interacting with the sortilin-related Vps10pdomain sorting receptor 2 (SorCS2). One of the downstream activity SorCS2 mediates is the downregulation of Rac activity, and therefore induces growth cone retraction [31]. The BDNF Val66Met polymorphism is able to engender neurodegenesis in two aspects, shrinkage of hippocampus and growth cone retraction, which can be responsible for pathogenesis of BD.

After long-patterned stimulus, the efficacy of neuronal synapses can be reduced in an activity-dependent manner. Long-term depression (LTD) is responsible for memory erasure, but also a bona fide learning and memory mechanism in the mammalian brain [41]. Bipolar disorder Patients suffer from cognitive decline [42], which can be a result of abnormal hippocampus LTD. Emerging lines of research reports that wild type BDNF pro-domain promotes LTD, whereas the Val66Met substitution inhibits hippocampus LTD [43]. Ninan et al. discovered that mice harboring the Val66Met substitution have deficiency in N-methyl-D-aspartate receptor (NMDAR)dependent plasticity [44]. Another line of research done by Mizui et al. demonstrates that the wild type BDNF propeptide induces endocytosis of GluA2, an underlying mechanism for LTD, which can be attenuated by Mettype pro-peptide [43]. One possible molecular mechanism for the attenuative effect of Met-type pro-peptide can be explained by the increased interaction of $m-B D N F$ and its pro-peptide. It has been proved that that BDNF propeptide binds to BDNF with high affinity and specificity [45]. With the Val to Met amino acid residue substitution, this interaction is strengthened in the entire acidic to neutral $\mathrm{pH}$ range [45]. The strong interaction caused by Met-type pro-peptide could be a mechanism for its deficiency in inducing LTD. The Val66Met polymorphism possibly accounts for cognitive decline in BD by interfering hippocampus LTD.

$\mathrm{BD}$ is characterized by episodes of mood alterations between mania and depression. If the episodes cycle rapidly, one can also suffer from mixed symptoms at both poles [46]. 5-15\% of BD patients have rapid cycling symptoms [47]. The BDNF Val66Met polymorphism is associated with rapid cycling episodes in BD. A familybased association study and haplotype analyses association study with 312 nuclear families done by Müller et al. confirmed this correlation. Participants with rapid cycling $\mathrm{BD}$ symptoms showed significant associations with the Val66Met polymorphism, while the non-rapid cycling group did not [48]. The aforementioned evidence indicates that the Val66Met polymorphism is not only reliable for the neurological dysfunctions in BD, but also a factor causing the mood swings between depressive and manic episodes.

\subsection{Arg125Met}

The Arg125Met polymorphism refers to an amino acid residue substitution on site 125 in the pro-domain of BDNF. This substitution is very near the cleavage site between BDNF pro-domain and $\mathrm{m}$-domain, indicating its potential in regulating the maturation of BDNF (Fig. 4). There are two basic assumptions for how the Arg125Met could cause BD, based on the fact that the substitution is harbored in the BDNF pro-domain: one being that this substitution may affect the interaction between pro-BDNF and its receptors; or it may affect the cleavage of proBDNF and also consequently the maturation of BDNF. Evidence demonstrating the substitution is not at the interaction site of pro-BDNF and its receptors suggests that the first hypothesis is less tenable. Up till now, the receptors Pro-BDNF interacts with on the synapses are p75NTR and sortilin receptor (Fig. 3). Pro-BDNF interacts with p75NTR, through its mature domain [49], excluding the possibility that the Arg $125 \mathrm{Met}$ affects proBDNF's binding with p75NTR. On the other hand, although pro-BDNF binds to the sortilin receptor with its pro-domain, a domain deletion study showing that the functional binding site is from 44 to 10350 , precludes the Arg125Met variant's impact on the binding specificity as well. Hence, the first hypothesis mentioned above is unlikely the mechanism the Arg125Met variant could cause neurological disorders.

The second hypothesis, however, is supported by experimental data. Kojima et al.'s study demonstrates that Arg125Met substitution interferes with pro-BDNF cleavage [35]. Hippocampal neurons transfected with the Arg 125Met variant produces only a residue amount of mBDNF both inside cell and in secretion. The ratio of proBDNF to $\mathrm{m}-\mathrm{BDNF}$ is also significantly higher than that of the control group, proving that the BDNF Arg124Met variant is cleavage-resistant [51]. Moreover, treatment with the BDNF Arg125Met variant induces cell apoptosis in cultured cerebellar granule, while m-BDNF promotes cell survival [51]. Therefore, the Arg125Met has the potential to regulate the quantity trade-off between proBDNF and $\mathrm{m}-\mathrm{BDNF}$, mediating neuronal apoptosis. We have discussed how $\mathrm{BD}$ can be considered as a neurodegenesis disease has been discussed, and here we propose a conjecture for a possible mechanism of the BDNF Arg125Met variant for BD pathogenesis. After binding with p75NTR or sortilin receptor in the synapse, pro-BDNF activates the NF- $\kappa \mathrm{B}$ signaling pathway that promotes cell survival, and the JNK signaling pathway that elicits cell apoptosis as well (Fig. 3) [9]. Meanwhile, $\mathrm{m}$-BDNF's binding with the TrkB receptor activates PI3K signaling pathway, which has an anti-apoptotic activity (Fig. 3) [9]. The cleavage resistant BDNF Arg125Met results in a higher ratio of pro-BDNF to m-BDNF, and therefore can cause neuronal loss by upregulating the JNK 
signal pathway and downregulating the PI3K signaling pathway. Nonetheless, pro-BDNF can also activate the $\mathrm{NF}-\kappa \mathrm{B}$ signaling pathway to promote cell survival, so it is possible for the patient to restore the balance at times and have a period of euthymia (Fig. 5).

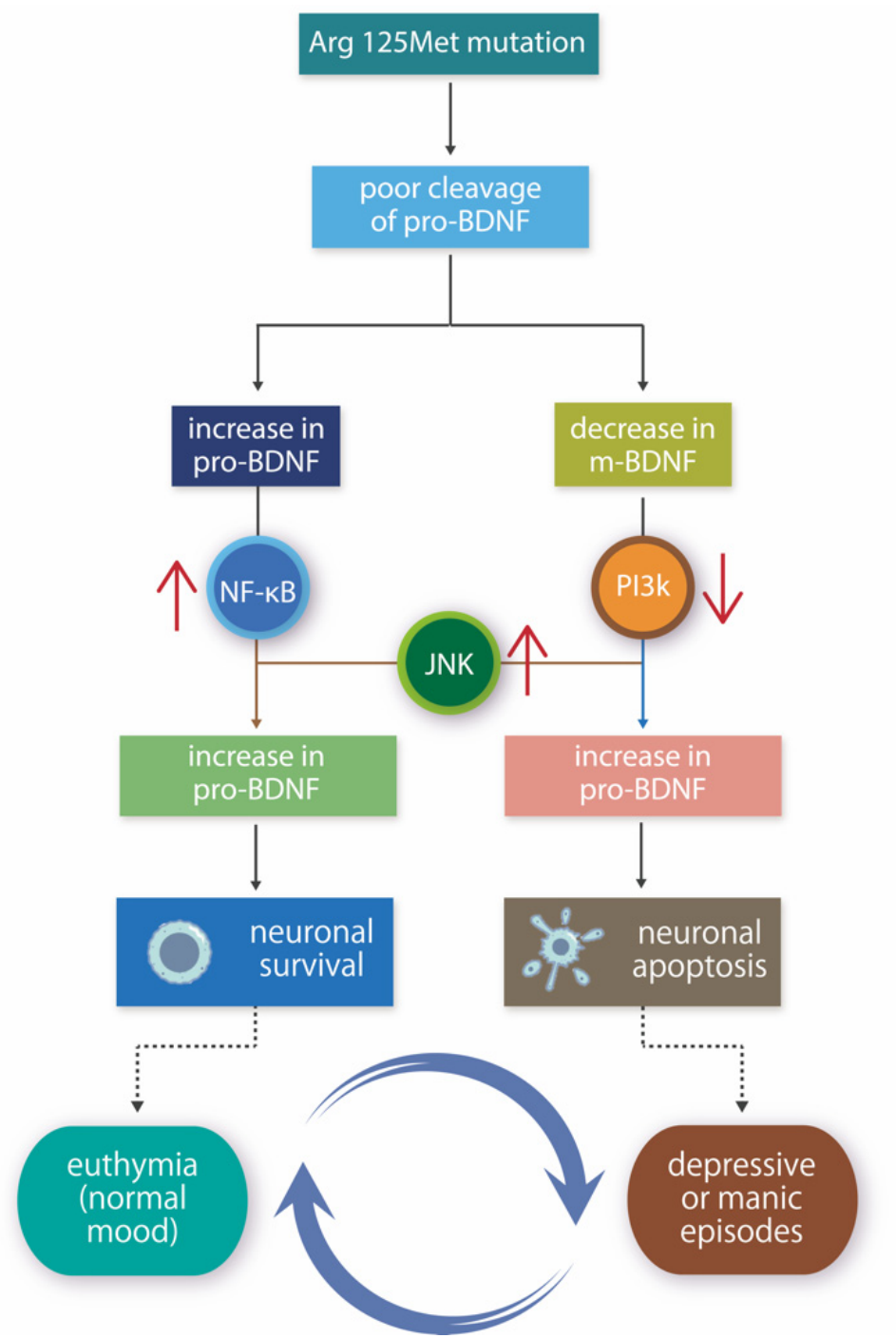

Fig 5. A hypothesis for how Arg125Met contributes to BD Pathogenesis. Poor cleavage of pro-BDNF results in an increase in pro-BDNF and a decrease in $\mathrm{m}-\mathrm{BDNF}$. Increase in Pro-BDNF upregulates both the NF- $\mathrm{KB}$ and the JNK signaling pathways (brown arrows). The NF- $\mathrm{KB}$ signaling pathway promotes cell survival while the JNK signaling pathway facilitates neuronal apoptosis. Decrease in m-BDNF downregulates PI3K signaling pathway (cyan arrows). PI3K signaling pathway has an antiapoptotic effect and its downregulation accelerates neuronal apoptosis. Neurodegenesis caused by neuronal apoptosis is a possible factor for causing BD symptoms. The patient may restore the balance between neuronal apoptosis and survival, having a period of euthymia at some point because the NF- $\mathrm{KB}$ signaling pathway promotes cell survival. Arrows with dashing lines represent mechanism that is not yet lucid.

Blue arrows at the bottom represent the cycling of euthymia and mood episodes in BD.

\section{Treatments}

\subsection{Existing Pharmacological Treatments of BD}

Over the past years, scientists have found various treatments for bipolar disorder. Our discussion focuses on pharmacotherapy including lithium and valproic acid (VPA) because of their extensiveness. A study has shown that lithium and valproic acid could protect neurons based on BDNF [52].

A third of patients can be successfully treated by lithium treatment53. Lithium treatment is an effective way to cure BD. The mechanism of lithium stabilized mood has been revealed recent years. The efficacy of lithium is inhibiting glycogen synthase kinase-3 $\beta$ (GSK3 $\beta$ ) which is similar to BDNF. In the treatment of $B D$, when GSK3 $\beta$ is active, glutamatergic synapses will be reduced. Then, neuronal excitability decreases which result in depressive mood. On the contrary, when the activation of GSK $3 \beta$ decreases, glutamatergic synapses increase. After which, neuronal excitability will increase and the symptoms of bipolar disorder could be relieved [53].

VPA is known to have effects on g-aminobutyric acid (GABA), which is important to the central nervous system of mammals and related to $\mathrm{BD}$ [52]. GABA is the most important inhibitor which acts through GABARs and 
influenced by the signaling pathways of BDNF [54]. GSK $3 \beta$ phosphorylates is the anchor protein for GABARs, which induces the formation of new GABAergic synapses [53]. VPA regular the metabolism of GABA through inhibiting the related enzymes. Thus, VAP achieves to cure BD [52].

Though traditional Pharmacotherapy is widely used, it still limited to causing drug resistance and side effects [55]. Long-term of pharmacological treatments give rise to drug resistance, leading to a decline in the efficacy of medicine. Another significant issue is side effects include liver and kidney damage. As well as hand shaking, diarrhea and nausea that make patients suffering a lot. Therefore, finding new therapeutic strategies is needed.

\subsection{CRISPR: as An Innovative Therapy Based On BDNF}

CRISPR/Cas9 system was first discovered in 1987 by Ishino. As an emerging technology, it exerted an extraordinary utility of altering target genes. The CRISPR/Cas9 system recognizes and binds the particular DNA sequence. Then, cut the target loci causing a double strand break (DSB) which will repaired by the cell DNA repaired mechanisms. Meanwhile, introduce new mutations into the sequence making it possible for altering target genes by Crispr-Cas9 system [56, 57].

Evidence supports CRISPR-Cas9 system's effectiveness in treating multiple neurological disorders. The exist research of releasing opioid addiction has mentioned CRISPR/Cas9 as a potential therapy with successful animal experiment [58]. The similar results have been demonstrated for the study of Alzheimer disease (AD). Thus, CRISPR-Cas9 system has showed feasibility of treating bipolar disorder by rescuing the mutated site of BDNF and regular signaling pathways [13].

Generating variant animals models is an important process of discover new treatment. Several animal experiments in BDNF have interesting findings. By generating variant BDNF mice (BDNFMet/Met) they found that the anxiety behaviors of mice could not be released by antidepressant [59]. However, extending the half life of producing BDNF or secreting more showed therapeutic improvement which indicated the potential of applying CRISPR therapy is feasible [59]. Another mice study showed reduction in BDNF causes neurogenesis damage [60].Depression symptoms in BD can be alleviated by increasing the secretion of BDNF. Thus, alter the mutation on BDNF could regular the mood of patients. These evidence illustrated CRISPR/Cas9, a novel approach of therapeutic strategies, stand a chance of releasing $\mathrm{BD}$.

Despite the development of CRISPR/Cas9 system, significant challenges to apply it into treatments remain, such as off target effects, difficulties in delivering to cells, and Ethical issues. The improvements of gene therapy is worth discussing. Here we will provide several possible solutions to these issues. We could design specific guide RNA to reduce off-target rate. Reducing the target sequence length and decreasing the duration of Cas9 nuclease action has been confirmed effective [61]. As for delivery, the most common one is micro-injection with high successful rate. Adeno-associated virus (AAV) is widely used vector whose efficiency depends on capacity. More efficient vectors are being discovered by scientists. The safety and permission of adapting gene editing technology is still worth to discuss. There is no settled opinion until now despite some interim criteria. Moreover, financial support of gene editing projects such as ELSI and SEED is important [62].

\section{Conclusion}

The Pathogenic mechanism remains unclear because of multiple factors of bipolar disorder that requires further investigation. In this review, we illustrated the relationship between BDNF and two mutations, Val66Met polymorphism and Arg125Met, as well as their impact on bipolar disorder which is important for finding out treatments. Therefore, recent research is focusing on figuring out the mechanism of bipolar disorder and targeting intervention. After which, we could establish animal model, and finally put into human clinical treatment. Now we have effective drug therapy, but gene therapy is still theoretical. The technology of gene editing is a limitation which needs to be improved to solve the existing problems, such as off-target and lack of efficient vectors. Further research on BDNF is requiring for a better understanding of $\mathrm{BD}$.

\section{References}

1. Marwaha, S.,DurraniA. and Singh, S. (2013). Employment outcomes in people with bipolar disorder: a systematic review. Acta Psychiatr. Scand. 128, 179-193.

2. Culpepper, L. (2014). The diagnosis and treatment of bipolar disorder: decision-making in primary care. The primary care companion for CNS disorders 16 .

3. Rowland, T. A. and Marwaha, S. (2018). Epidemiology and risk factors for bipolar disorder. Therapeutic advances in psychopharmacology 8, 251-269.

4. Grande, I.,Fries, G. R.,Kunz, M. and Kapczinski, F. (2010). The Role of BDNF as a Mediator of Neuroplasticity in Bipolar Disorder. Psychiatry Investig 7, 243-250.

5. Cunha, A. B. M.,Frey, B. N.,Andreazza, A. C., Goi, J. D.,Rosa, A. R.,Gonçalves, C. A.,Santin, A. and Kapczinski, F. (2006). Serum brain-derived neurotrophic factor is decreased in bipolar disorder during depressive and manic episodes. Neurosci. Lett. 398, 215-219.

6. Hofer, M.,Pagliusi, S. R.,Hohn, A.,Leibrock, J. and Barde, Y. (1990). Regional distribution of brainderived neurotrophic factor mRNA in the adult mouse brain. The EMBO journal 9, 2459-2464. 
7. Panja, D. and Bramham, C. R. (2014). BDNF mechanisms in late LTP formation: a synthesis and breakdown. Neuropharmacology 76, 664-676.

8. Notaras, M. and van den Buuse, M. (2019). Brainderived neurotrophic factor (BDNF): novel insights into regulation and genetic variation. The Neuroscientist 25, 434-454.

9. Kowiański, P.,Lietzau, G.,Czuba, E.,Waśkow, M.,Steliga, A. and Moryś, J. (2018). BDNF: a key factor with multipotent impact on brain signaling and synaptic plasticity. Cell. Mol. Neurobiol. 38, 579593.

10. Karege, F.,Bondolfi, G.,Gervasoni, N.,Schwald, M.,Aubry, J.-M. and Bertschy, G. (2005). Low brainderived neurotrophic factor (BDNF) levels in serum of depressed patients probably results from lowered platelet BDNF release unrelated to platelet reactivity. Biol. Psychiatry 57, 1068-1072.

11. Polyakova, M.,Stuke, K.,Schuemberg, K.,Mueller, K.,Schoenknecht, P. and Schroeter, M. L. (2015). BDNF as a biomarker for successful treatment of mood disorders: A systematic \& quantitative metaanalysis. J. Affect. Disord. 174, 432-440.

12. Wu, R.,Fan, J.,Zhao, J.,Calabrese, J. R. and Gao, K. (2014). The relationship between neurotrophins and bipolar disorder. Expert Rev. Neurother. 14, 51-65.

13. Harrison, P. J.,Cipriani, A.,Harmer, C. J.,Nobre, A. C.,Saunders, K.,Goodwin, G. M. and Geddes, J. R. (2016). Innovative approaches to bipolar disorder and its treatment. Ann. N. Y. Acad. Sci. 1366, 76-89.

14. Merikangas, K. R.,Jin, R.,He, J.-P.,Kessler, R. C.,Lee, S.,Sampson, N. A.,Viana, M. C.,Andrade, L. H.,Hu, C.,Karam, E. G.,Ladea, M.,Medina-Mora, M. E.,Ono, Y.,Posada-Villa, J.,Sagar, R.,Wells, J. E. and Zarkov, Z. (2011). Prevalence and Correlates of Bipolar Spectrum Disorder in the World Mental Health Survey Initiative. Arch. Gen. Psychiatry 68, 241-251.

15. Tsuchiya, K. J.,Byrne, M. and Mortensen, P. B. (2003). Risk factors in relation to an emergence of bipolar disorder: a systematic review. Bipolar Disord. 5, 231-242.

16. Marty, M. and Segal, D. (2015). DSM-5: Diagnostic and Statistical Manual of Mental Disorders. 965-970.

17. Yatham, L. N.,Kennedy, S. H.,Parikh, S. V.,Schaffer, A.,Beaulieu, S.,Alda, M.,O’Donovan, C.,MacQueen, G.,McIntyre, R. S.,Sharma, V.,Ravindran, A., Young, L. T.,Milev, R.,Bond, D. J.,Frey, B. N.,Goldstein, B. I.,Lafer, B.,Birmaher, B.,Ha, K.,Nolen, W. A. and Berk, M. (2013). Canadian Network for Mood and Anxiety Treatments (CANMAT) and International Society for Bipolar Disorders (ISBD) collaborative update of CANMAT guidelines for the management of patients with bipolar disorder: update 2013 . Bipolar Disord. 15, 1-44.

18. Hamdani, N.,Daban-Huard, C.,Lajnef, M.,Gadel, R.,Le Corvoisier, P.,Delavest, M.,Carde, S.,Lépine, J.-P.,Jamain, S.,Houenou, J.,Galeh, B.,Richard, J.R.,Aoki, M.,Charron, D.,Krishnamoorthy, R., Yolken, R.,Dickerson, F.,Tamouza, R. and Leboyer, M.
(2015). Cognitive deterioration among bipolar disorder patients infected by Toxoplasma gondii is correlated to interleukin 6 levels. J. Affect. Disord. $179,161-166$.

19. Townsend, J. and Altshuler, L. L. (2012). Emotion processing and regulation in bipolar disorder: a review. Bipolar Disord. 14, 326-339.

20. Newberg, A. R.,Catapano, L. A.,Zarate, C. A. and Manji, H. K. (2008). Neurobiology of bipolar disorder. Expert Rev. Neurother. 8, 93-110.

21. Maletic, V. and Raison, C. (2014). Integrated Neurobiology of Bipolar Disorder. Frontiers in Psychiatry 5, 98.

22. Hashimoto, K. (2010). Brain-derived neurotrophic factor as a biomarker for mood disorders: An historical overview and future directions. Psychiatry Clin. Neurosci. 64, 341-357.

23. Bath, K. G. and Lee, F. S. (2006). Variant BDNF (Val66Met) impact on brain structure and function. Cognitive, Affective, \& Behavioral Neuroscience 6, 79-85.

24. Duman, R. S. and Monteggia, L. M. (2006). A Neurotrophic Model for Stress-Related Mood Disorders. Biol. Psychiatry 59, 1116-1127.

25. Green, E. and Craddock, N. (2003). Brain-derived neurotrophic factor as a potential risk locus for bipolar disorder: Evidence, limitations, and implications. Curr. Psychiatry Rep. 5, 469-476.

26. Fernandes, B. S.,Molendijk, M. L.,Köhler, C. A.,Soares, J. C.,Leite, C. M. G. S.,Machado-Vieira, R.,Ribeiro, T. L.,Silva, J. C.,Sales, P. M. G.,Quevedo, J.,Oertel-Knöchel, V.,Vieta, E.,González-Pinto, A.,Berk, M. and Carvalho, A. F. (2015). Peripheral brain-derived neurotrophic factor (BDNF) as a biomarker in bipolar disorder: a meta-analysis of 52 studies. BMC Med. 13, 289.

27. Kim, H.-W.,Rapoport, S. I. and Rao, J. S. (2010). Altered expression of apoptotic factors and synaptic markers in postmortem brain from bipolar disorder patients. Neurobiol. Dis. 37, 596-603.

28. Teng, H. K.,Teng, K. K.,Lee, R.,Wright, S., Tevar, S.,Almeida, R. D.,Kermani, P.,Torkin, R.,Chen, Z.Y.,Lee, F. S.,Kraemer, R. T.,Nykjaer, A. and Hempstead, B. L. (2005). ProBDNF Induces Neuronal Apoptosis via Activation of a Receptor Complex of p75\&lt;sup\&gt;NTR\&lt;/sup\&gt; and Sortilin. The Journal of Neuroscience 25, 5455.

29. Lindholm, J. S. O. and Castrén, E. (2014). Mice with altered BDNF signaling as models for mood disorders and antidepressant effects. Front. Behav. Neurosci. 8.

30. Teng, H. K., Teng, K. K.,Lee, R.,Wright, S., Tevar, S.,Almeida, R. D.,Kermani, P.,Torkin, R.,Chen, Z.Y.,Lee, F. S.,Kraemer, R. T.,Nykjaer, A. and Hempstead, B. L. (2005). ProBDNF Induces Neuronal Apoptosis via Activation of a Receptor Complex of p75 and Sortilin. The Journal of Neuroscience 25, 5455. 
31. Anastasia, A.,Deinhardt, K.,Chao, M. V.,Will, N. E.,Irmady, K.,Lee, F. S.,Hempstead, B. L. and Bracken, C. (2013). Val66Met polymorphism of BDNF alters prodomain structure to induce neuronal growth cone retraction. Nat. Commun. 4, 2490.

32. Reichardt, L. F. (2006). Neurotrophin-regulated signalling pathways. Philos. Trans. R. Soc. Lond. B. Biol. Sci. 361, 1545-1564.

33. Notaras, M. and van den Buuse, M. (2020). Neurobiology of BDNF in fear memory, sensitivity to stress, and stress-related disorders. Mol. Psychiatry 25, 2251-2274.

34. Finn, R. D.,Coggill, P.,Eberhardt, R. Y.,Eddy, S. R.,Mistry, J.,Mitchell, A. L.,Potter, S. C.,Punta, M.,Qureshi, M. and Sangrador-Vegas, A. (2016). The Pfam protein families database: towards a more sustainable future. Nucleic Acids Res. 44, D279D285.

35. Egan, M. F.,Kojima, M.,Callicott, J. H.,Goldberg, T. E.,Kolachana, B. S.,Bertolino, A.,Zaitsev, E.,Gold, B.,Goldman, D. and Dean, M. (2003). The BDNF val66met polymorphism affects activity-dependent secretion of BDNF and human memory and hippocampal function. Cell 112, 257-269.

36. Lu, R.-B.,Chen, S.-L.,Lee, S.-Y.,Chang, Y.-H.,Chen, S.-H.,Chu, C.-H.,Tzeng, N.-S.,Lee, I. H.,Chen, P. S.,Yeh, T. L.,Huang, S.-Y.,Yang, Y. K. and Hong, J.S. (2012). Neuroprotective and neurogenesis agent for treating bipolar II disorder: Add-on memantine to mood stabilizer works. Med. Hypotheses 79, 280283.

37. Lim, Y. Y.,Villemagne, V. L.,Laws, S. M.,Ames, D.,Pietrzak, R. H.,Ellis, K. A.,Harrington, K. D.,Bourgeat, P.,Salvado, O. and Darby, D. (2013). BDNF Val66Met, $A \beta$ amyloid, and cognitive decline in preclinical Alzheimer's disease. Neurobiol. Aging 34, 2457-2464.

38. Matsuo, K.,Walls-Bass, C. M.,Nicoetti, M. A.,Diershke, N.,Hatch, J. P.,Nery, F. G.,Frey, B. N.,Monkul, E. S.,Zunta, G. B. and Bowden, C. L. (2007). Brain-derived neurotrophic factor val66met polymorphism and morphometric abnormality in bipolar disorder patients. Biol. Psychiatry 61, 141S$141 \mathrm{~S}$.

39. Chen, Z.-Y.,Jing, D.,Bath, K. G.,Ieraci, A.,Khan, T.,Siao, C.-J.,Herrera, D. G.,Toth, M., Yang, C. and McEwen, B. S. (2006). Genetic variant BDNF (Val66Met) polymorphism alters anxiety-related behavior. Science 314, 140-143.

40. Gonzalez-Billault, C.,Muñoz-Llancao, P.,Henriquez, D. R.,Wojnacki, J.,Conde, C. and Caceres, A. (2012). The role of small GTPases in neuronal morphogenesis and polarity. Cytoskeleton 69, 464485.

41. Connor, S. A. and Wang, Y. T. (2015). A Place at the Table: LTD as a Mediator of Memory Genesis. The Neuroscientist 22, 359-371.
42. Berridge, M. J. (2014). Calcium signalling and psychiatric disease: bipolar disorder and schizophrenia. Cell Tissue Res. 357, 477-492.

43. Mizui, T.,Ishikawa, Y.,Kumanogoh, H.,Lume, M.,Matsumoto, T.,Hara, T.,Yamawaki, S.,Takahashi, M.,Shiosaka, S. and Itami, C. (2015). BDNF propeptide actions facilitate hippocampal LTD and are altered by the common BDNF polymorphism Val66Met. Proceedings of the National Academy of Sciences 112, E3067-E3074.

44. Ninan, I.,Bath, K. G.,Dagar, K.,Perez-Castro, R.,Plummer, M. R.,Lee, F. S. and Chao, M. V. (2010). The BDNF Val66Met polymorphism impairs NMDA receptor-dependent synaptic plasticity in the hippocampus. J. Neurosci. 30, 8866-8870.

45. Uegaki, K.,Kumanogoh, H.,Mizui, T.,Hirokawa, T.,Ishikawa, Y. and Kojima, M. (2017). BDNF binds its pro-peptide with high affinity and the common Val66Met polymorphism attenuates the interaction. Int. J. Mol. Sci. 18, 1042.

46. Vieta, E.,Berk, M.,Schulze, T. G.,Carvalho, A. F.,Suppes, T.,Calabrese, J. R.,Gao, K.,Miskowiak, K. W. and Grande, I. (2018). Bipolar disorders. Nature Reviews Disease Primers 4, 18008.

47. Bell, C. C. (1994). DSM-IV: Diagnostic and Statistical Manual of Mental Disorders. JAMA 272, 828-829.

48. Müller, D. J.,De Luca, V.,Sicard, T.,King, N.,Strauss, J. and Kennedy, J. L. (2006). Brain-derived neurotrophic factor (BDNF) gene and rapid-cycling bipolar disorder: family-based association study. The British Journal of Psychiatry 189, 317-323.

49. Teng, H. K.,Teng, K. K.,Lee, R.,Wright, S.,Tevar, S.,Almeida, R. D.,Kermani, P.,Torkin, R.,Chen, Z.Y. and Lee, F. S. (2005). ProBDNF induces neuronal apoptosis via activation of a receptor complex of p75NTR and sortilin. J. Neurosci. 25, 5455-5463.

50. Chen, Z.-Y.,Ieraci, A.,Teng, H.,Dall, H.,Meng, C.X.,Herrera, D. G.,Nykjaer, A.,Hempstead, B. L. and Lee, F. S. (2005). Sortilin controls intracellular sorting of brain-derived neurotrophic factor to the regulated secretory pathway. J. Neurosci. 25, 61566166.

51. Koshimizu, H.,Kiyosue, K.,Hara, T.,Hazama, S.,Suzuki, S.,Uegaki, K.,Nagappan, G.,Zaitsev, E.,Hirokawa, T.,Tatsu, Y.,Ogura, A.,Lu, B. and Kojima, M. (2009). Multiple functions of precursor $\mathrm{BDNF}$ to $\mathrm{CNS}$ neurons: negative regulation of neurite growth, spine formation and cell survival. Mol. Brain 2, 27.

52. Chiu, C.-T.,Wang, Z.,Hunsberger, J. G. and Chuang, D.-M. (2013). Therapeutic potential of mood stabilizers lithium and valproic acid: beyond bipolar disorder. Pharmacol. Rev. 65, 105-142.

53. Sato, K. (2021). Why is lithium effective in alleviating bipolar disorder? Med. Hypotheses 147, 110484. 
54. Agrawal, R.,Kalmady, S. V. and Venkatasubramanian, G. (2017). In SilicoModeldriven Assessment of the Effects of Brain-derived Neurotrophic Factor Deficiency on Glutamate and Gamma-Aminobutyric Acid: Implications for Understanding Schizophrenia Pathophysiology. Clinical psychopharmacology and neuroscience : the official scientific journal of the Korean College of Neuropsychopharmacology 15, 115-125.

55. Hui Poon, S.,Sim, K. and Baldessarini, R. J. (2015). Pharmacological Approaches for Treatment-resistant Bipolar Disorder. Current neuropharmacology 13, 592-604.

56. Li, H.,Yang, Y.,Hong, W.,Huang, M.,Wu, M. and Zhao, X. (2020). Applications of genome editing technology in the targeted therapy of human diseases: mechanisms, advances and prospects. Signal transduction and targeted therapy 5, 1-1.

57. Munawar, N. and Ahmad, A. (2021). CRISPR/Cas System: An Introduction. CRISPR Crops: The Future of Food Security, 1-35.

58. Wang, S.-C.,Chen, Y.-C.,Lee, C.-H. and Cheng, C.M. (2019). Opioid Addiction, Genetic Susceptibility, and Medical Treatments: A Review. Int. J. Mol. Sci. 20, 4294.

59. Chen, Z.,Jing, D.,Bath, K.,Ieraci, A.,Khan, T.,Siao, C.,Herrera, D.,Toth, M. and Yang, C.). McEwen BSet al (2006) Genetic variant BDNF (Val66Met) polymorphism alters anxiety-related behavior. Science 314, 140-143.

60. Arosio, B.,Guerini, F. R.,Voshaar, R. C. O. and Aprahamian, I. (2021). Blood Brain-Derived Neurotrophic Factor (BDNF) and Major Depression: Do We Have a Translational Perspective? Front. Behav. Neurosci. 15, 626906-626906.

61. Naeem, M.,Majeed, S.,Hoque, M. Z. and Ahmad, I. (2020). Latest Developed Strategies to Minimize the Off-Target Effects in CRISPR-Cas-Mediated Genome Editing. Cells 9, 1608.

62. Howard, H. C.,van El, C. G.,Forzano, F.,Radojkovic, D.,Rial-Sebbag, E.,de Wert, G.,Borry, P.,Cornel, M. C.,Public and Professional Policy Committee of the European Society of Human, G. (2018). One small edit for humans, one giant edit for humankind? Points and questions to consider for a responsible way forward for gene editing in humans. European journal of human genetics : EJHG 26, 1-11. 